\title{
THE $X$-VARIETIES FOR CR MAPPINGS BETWEEN HYPERQUADRICS*
}

\author{
JOHN P. D'ANGELO ${ }^{\dagger}$ \\ Dedicated to Salah Baouendi as his seventieth birthday approaches
}

Key words. CR mappings, hermitian forms, unit sphere, hyperquadrics, proper holomorphic mappings, X-variety

AMS subject classifications. 32V15, 32H35, 32H99, 32L05

0. Introduction. Let $M$ and $M^{\prime}$ denote smooth CR submanifolds of complex Euclidean spaces of possibly different dimensions. One of the most basic problems in CR geometry is to relate properties of the collection of smooth CR mappings $f$ : $M \rightarrow M^{\prime}$ to the geometries of $M$ and $M^{\prime}$. See the monograph [BER] for the complete theory of CR mappings and references.

Perhaps the simplest question about CR mappings is whether, given $M$ and $M^{\prime}$, there exist any nonconstant smooth CR mappings between $M$ and $M^{\prime}$. For spheres and hyperquadrics there are many examples; as the target dimension increases the dimension of the moduli space of examples is unbounded above. A CR mapping $f$ is a solution of a first order system of partial differential equations, but the information that $f(M) \subset M$ is a nonlinear condition on $f$. When $M$ and $M^{\prime}$ are spheres or hyperquadrics, the approach in this paper eliminates the nonlinear aspect of the problem.

This paper considers CR mappings between hyperquadrics, including spheres as a special case. We are interested in the structure of CR mappings from a given hyperquadric to hyperquadrics in all possible target dimensions. Placing restrictions on the dimension of the target and on the signature then places restrictions on the possible mappings. In Section 1 we study a certain variety $X_{f}$ naturally associated with a holomorphic mapping $f$ such that $f(M) \subset M^{\prime}$, and we use it to partially address the issue of the complexity of rational mappings taking $M$ to $M^{\prime}$. Homogenization plays a crucial role in our study; the variety $X_{f}$ is easier to understand when $f$ is homogeneous. In Section 2 we further analyze the homogeneous case, generalizing work done by the author for spheres [D2], [D3].

The complexity question is interesting and quite difficult even when $M$ and $M^{\prime}$ are spheres. Write $\|z\|^{2}$ for the squared Euclidean norm in all dimensions. Let $M$ denote the unit sphere given by $\left\{z:\|z\|^{2}=1\right\}$ in $\mathbf{C}^{n}$ and $M^{\prime}$ the unit sphere in $\mathbf{C}^{N}$. For $n \geq 2$, Forsteneric [F1] proved that a smooth (infinitely differentiable) CR mapping between spheres must be the restriction of a rational mapping to the sphere. See also [CS]. The degree of a rational mapping provides one measure of its complexity.

When $1=n \leq N$, there is no restriction on the degree of a rational mapping sending the circle to a sphere. For $n \geq 2$, Forstneric [F1] also found a crude bound on the degree of the rational mapping (from sphere to sphere) in terms of the dimensions

\footnotetext{
*Received June 30, 2006; accepted for publication February 2, 2007.

$\dagger$ Department of Mathematics, University of Illinois, 1409 W. Green St., Urbana, IL 61801, USA (jpda@math.uiuc.edu).
} 
$n$ and $N$. A sharp bound is not known. When $N<n$, the only smooth CR mappings between $M$ and $M^{\prime}$ are constant, and thus of degree 0 . When $n \leq N \leq 2 n-2$, the only smooth CR mappings between $M$ and $M^{\prime}$ are linear fractional transformations [Fa1], and hence of degree at most 1 . When $N=2 n-1$ there are mappings of degree 2 , and for $n \geq 3$ and $N=2 n-1$, the degree is at most 2 . When $n=2$ and $3=N=2 n-1$, the maximum degree is 3 . [Fa2]. When $n=2$, and the components of $f$ are assumed to be monomials, the degree is at most $2 N-3$, and this result is sharp. [DKR]. See also [D3], [F2], [H] , and [HJ] for related results concerning mappings between spheres and see [BH], [EHZ1], and [EHZ2] for generalizations to mappings between hyperquadrics.

Rational CR mappings between spheres become more complicated when the target dimension $N$ increases while the domain dimension $n$ remains fixed. When $n$ is fixed, for example, the degree of possible rational examples tends to infinity as $N$ does. The author ([D3]), based on work with Catlin ([CD1], [CD2]) proved the following result which strongly illustrates how the complexity of possible mappings grows with $N$.

TheOREM 0. Let $f=\frac{p}{q}$ be a rational mapping from $\mathbf{C}^{n}$ (minus the zero set of q) to $\mathbf{C}^{K}$ such that $\|f(z)\|^{2}<1$ when $\|z\|^{2} \leq 1$. Then there exist an integer $L$ and a polynomial $g: \mathbf{C}^{n} \rightarrow \mathbf{C}^{L}$ such that

$$
\left\|\frac{p \oplus g}{q}(z)\right\|^{2}=1
$$

when $\|z\|^{2}=1$.

In particular the dimension of the moduli space of smooth CR mappings between spheres tends to infinity as the target dimension tends to infinity. If we allow the target dimension to be arbitrary, then we can do almost whatever we want as long as we stay in the rational category. For a fixed target dimension the degree of $f$ is controlled (although establishing a sharp bound is an open problem) by the dimension of the target sphere.

The following simple examples reveal a basic difference between the sphere and other hyperquadrics, by showing that even more examples are possible. Let $Q(a, b)$ denote the hyperquadric in $\mathbf{C}^{a+b}$ defined by the equation

$$
\left|z_{1}\right|^{2}+\ldots+\left|z_{a}\right|^{2}-\left|z_{a+1}\right|^{2}-\ldots-\left|z_{a+b}\right|^{2}=1 .
$$

In this notation the sphere $S^{2 n-1}$ is also the hyperquadric $Q(n, 0)$. We give two examples involving an arbitrary holomorphic function $h$ on $\mathbf{C}^{2}$. Define $f: \mathbf{C}^{2} \rightarrow \mathbf{C}^{4}$ by

$$
f\left(z_{1}, z_{2}\right)=\left(z_{1}, h\left(z_{1}, z_{2}\right), z_{2}, h\left(z_{1}, z_{2}\right)\right) .
$$

It is evident that $f(Q(1,1)) \subset Q(2,2)$. Next define $g: \mathbf{C}^{2} \rightarrow \mathbf{C}^{4}$ by

$$
g\left(z_{1}, z_{2}\right)=\left(1, z_{1} h\left(z_{1}, z_{2}\right), z_{2} h\left(z_{1}, z_{2}\right), h\left(z_{1}, z_{2}\right)\right) .
$$

Then $g(Q(2,0)) \subset Q(3,1)$. In each case we cannot control the complexity of the mapping by some measure such as the degree of a rational function, because $h$ is arbitrary. The examples have one difference. The mapping $f$ has linearly dependent components, and hence the phenomenon (the lack of control of $h$ ) seems to be an artifact of linear dependence. The mapping $g$, however, has linearly independent components (unless $h$ is constant), and yet we still have no control on $h$. 
In each of these two cases, the number of negative eigenvalues in the defining equation of the target hyperquadric exceeds the number of negative eigenvalues in the defining equation of the domain hyperquadric. See $[\mathrm{BH}]$ for a super-rigidity result for mappings between hyperquadrics when this number is not allowed to increase. One could also eliminate such mappings from consideration by placing additional geometric assumptions such as transversality conditions on the mappings allowed.

Some general properties of mappings between hyperquadrics do closely mimic those of mappings between spheres. In Theorem 2 from Section 2 we find all homogeneous polynomial mappings (with minimal target dimension) between hyperquadrics. We also provide a simple method for generating polynomial mappings between hyperquadrics. This method plays a key role in the study of polynomial mappings between spheres. See [D1, D2, D3].

In this paper we will approach the issue of complexity by studying a certain variety $X_{f}$ associated with a holomorphic mapping $f$ which restricts to a CR mapping between real-analytic hypersurfaces. For spheres this variety plays an important role in Forstneric's proof of rationality [F1]. An efficient method for computing $X_{f}$ for spheres appears in [D4] and is recalled and generalized in this paper.

Let $M$ and $M^{\prime}$ be real-analytic real hypersurfaces in complex Euclidean spaces, with local defining equations $\rho=0$ near a point $p \in M$ and $\rho^{\prime}=0$ near a point $p^{\prime} \in M^{\prime}$. Let $f$ be a holomorphic mapping, defined in a neighborhood of $p$, with $f(p)=p^{\prime}$, such that $f(M) \subset M^{\prime}$. The definition of $X_{f}$ will be given in terms of $\rho$ and $\rho^{\prime}$; the form of the definition makes it obvious that $X_{f}$ does not depend on the choice of defining equations.

Suppose $f$ is holomorphic and $f(M) \subset M^{\prime}$. We then have the crucial equation

$$
\rho^{\prime}(f(z), \overline{f(z)})=0 \quad \text { when } \quad \rho(z, \bar{z})=0 .
$$

Polarizing (5) as usual we obtain

$$
\rho^{\prime}(f(z), \overline{f(w)})=0 \quad \text { when } \quad \rho(z, \bar{w})=0 .
$$

Many authors have used (6) to provide reflection principles. See for example [DF], [DW], [La], [P], and [W]. The variety $X_{f}$ is obtained by generalizing (6).

Definition of $X_{f}$. Let $M \subset \mathbf{C}^{n}$ and $M^{\prime} \subset \mathbf{C}^{N}$ be real-analytic real hypersurfaces, and let $U$ be a neighborhood of $p \in M$. Suppose $f: U \subset \mathbf{C}^{n} \rightarrow \mathbf{C}^{N}$ is holomorphic and $f(M) \subset M^{\prime}$. Let $\rho$ and $\rho^{\prime}$ be local defining equations near $p$ and $f(p)$. The $X$-variety $X_{f}$ is the subset of $\operatorname{Domain}(f) \times \operatorname{Target}(f)$ defined by

$$
X_{f}=\left\{(w, \zeta): \rho^{\prime}(f(z), \bar{\zeta})=0 \quad \text { when } \quad \rho(z, \bar{w})=0\right\} .
$$

By (6) it follows that $X_{f}$ contains the graph of $f$. In general $X_{f}$ is a larger set; we can use properties of $X_{f}$ to obtain some understanding of the complexity of $f$.

We note one subtle point before we go on. When $M$ is a hyperquadric, $\rho(z, \bar{w})=$ $\langle z, w\rangle-1$, where $\langle$,$\rangle denotes a sesquilinear Hermitian form. The condition \langle z, w\rangle=1$ is vacuous when $w=0$, and thus pairs $(0, \zeta)$ are not included in $X_{f}$. It seems that they should be included, and therefore one should extend the definition of $X_{f}$ to include 0 . The idea amounts to including the hyperplane at infinity when one works projectively. The formula in Theorem 1 for $X_{f}$ makes sense when $w=0$. At the end of Section 1 we use Theorem 1 to define the extended variety $Y_{f}$ so as to include the case $w=0$. 
Then $Y_{f}$ is an algebraic variety naturally associated with $f$, and $Y_{f}$ contains the graph of $f$.

Let $\mathcal{L}\left(\mathbf{C}^{N}, H(n, d)\right)$ denote the vector space of linear transformations from $\mathbf{C}^{N}$ to the space of homogeneous polynomials of degree $d$ in $n$ variables. Theorem 1 , our main result, exhibits a polynomial mapping $w \mapsto C(w) \in \mathcal{L}\left(\mathbf{C}^{N}, H(n, d)\right)$ which determines $X_{f}$. The matrix of $C(w)$ can be computed explicitly and easily, thus making Theorem 1 easy to use.

THEOREM 1. Let $\langle$,$\rangle denote both a nondegenerate Hermitian form on \mathbf{C}^{n}$ and one on $\mathbf{C}^{N}$. Let $M$ denote the hyperquadric in $\mathbf{C}^{n}$ defined by $\{z:\langle z, z\rangle=1\}$, and let $M^{\prime}$ be the hyperquadric in $\mathbf{C}^{N}$ defined by $\{\zeta:\langle\zeta, \zeta\rangle=1\}$. Let $f$ be a rational function such that $f(M) \subset M^{\prime}$. Let d denote the degree of the numerator of $f$ when $f$ is reduced to lowest terms. Then there is a polynomial mapping $w \mapsto C(w) \in \mathcal{L}\left(\mathbf{C}^{N}, H(n, d)\right)$ such that

$$
X_{f}=\{(w, \zeta): \zeta=f(w)+\operatorname{Kernel}(C(w))\}
$$

An immediate corollary of Theorem 1 is that $X_{f}$ is an affine space. A corollary of the proof is that $X_{f}$ is an affine bundle whenever $f$ is a homogeneous polynomial mapping. We provide examples to show, even in the polynomial case, that $X_{f}$ need not be the graph of $f$, need not be a bundle, and so on. We also provide examples where the kernel of $C(w)$ is trivial for all $w$, and hence $X_{f}$ is the graph of $f$, even though $f$ itself is rather complicated. We also compute $X_{f}$ and $Y_{f}$ for some mappings invariant under finite unitary groups.

In Section 2 we consider homogeneous mappings. We determine the homogeneous polynomial mappings between hyperquadrics. We see in particular, for any homogeneous polynomial mapping of degree $d$ mapping a hyperquadric to a hyperquadric, that the coefficient vectors of the monomials of degree $d$ are linearly independent. This result provides the minimum target dimension for such maps. See Theorem 2 and Corollary 5. We provide an elementary procedure for writing down many mappings between hyperquadrics. In the special case of spheres we show that the homogeneous mappings can be characterized as extremals in terms of the volumes of proper holomorphic images of balls.

This paper is dedicated to Salah Baouendi, whose work in CR Geometry has profoundly influenced the author in many ways. He would also like to acknowledge Franc Forstneric and Peter Ebenfelt for useful comments on ideas related to this paper.

The author acknowledges support from NSF grant DMS 05-00765.

1. Computation of the $X$-variety for mappings between hyperquadrics. Let $M$ and $M^{\prime}$ denote real-analytic hypersurfaces. We recall from the introduction the definition of $X_{f}$ when $f$ is a holomorphic mapping which maps $M$ into $M^{\prime}$.

Definition 1. Let $U$ be a neighborhood of $p \in \mathbf{C}^{n}$. Suppose $f: U \subset \mathbf{C}^{n} \rightarrow \mathbf{C}^{N}$ is holomorphic and $f(M) \subset M^{\prime}$. Let $\rho$ and $\rho^{\prime}$ be local defining equations near $p$ and $f(p)$. The $X$-variety $X_{f}$ is the subset of $\operatorname{Domain}(f) \times \operatorname{Target}(f)$ defined by

$$
X_{f}=\left\{(w, \zeta): \rho^{\prime}(f(z), \bar{\zeta})=0 \quad \text { when } \quad \rho(z, \bar{w})=0\right\} .
$$

To understand this definition and to anticipate Theorem 1 , we begin by calculat$\operatorname{ing} X_{L}$ for a linear mapping from $\mathbf{C}^{n}$ to $\mathbf{C}^{N}$ such that $\|L(z)\|=1$ when $\|z\|=1$. 
Such an $L$ must be injective. Since the dimensions need not be equal, $L$ is not necessarily unitary. In Example $1\langle$,$\rangle denotes the usual Euclidean inner product, although$ afterwards we will let $\langle$,$\rangle denote an arbitrary nondegenerate, not necessarily positive$ definite, Hermitian form.

Example 1. ([D4]). $X_{L}=\left\{(w, \zeta): \zeta=L(w)+\operatorname{Kernel}\left(L^{*}\right)\right\}$. To verify this fact, combine (9) and (6) using $\rho^{\prime}=\langle\rangle-$,1 to obtain

$$
X_{L}=\{(w, \zeta):\langle L(z), \zeta-L(w)\rangle=0 \text { when }\langle z, w\rangle=1\} .
$$

Introducing the adjoint $L^{*}$ we obtain

$$
X_{L}=\left\{(w, \zeta):\left\langle z, L^{*}(\zeta-L(w))\right\rangle=0 \text { when }\langle z, w\rangle=1\right\} .
$$

Since both equations in (11) are homogeneous in $z,(11)$ can hold only if its first equation holds for all $z$. Hence for $w \neq 0,(w, \zeta) \in X_{L}$ if and only if

$$
L^{*}(\zeta-L(w))=0
$$

Formula (12) is the famous equation for least squares regression, arising from seeking the minimum distance of a given vector to the image of $L$. In our context (12) shows that $X_{L}$ is an affine bundle. For each $w$, the fibre over $w$ is $L(w)+\operatorname{Kernel}\left(L^{*}\right)$; the dimension does not vary with $w$, Furthermore, since $L^{*}$ is injective here if and only if $L$ is equi-dimensional and unitary, it follows that $X_{L}$ equals the graph of $L$ if and only if $L$ is an equi-dimensional mapping. The fibre dimension of $X_{L}$ measures precisely the excess in target dimension.

In Example 1 it is natural to define the fibre over $w=0$ also to be the kernel of $L^{*}$. This comment presages Definition 2 of the extended variety $Y_{L}$. We see, for all $(w, \zeta)$, that $(w, \zeta) \in Y_{L}$ if and only if (12) holds.

The ideas of Example 1 generalize significantly. The main difference will be that the fibre over $w$ will change from point to point. We will compute $X_{f}$ and study its properties whenever $f$ is a rational mapping between non-degenerate hyperquadrics. Thus we write $\langle$,$\rangle to denote a nondegenerate Hermitian form on \mathbf{C}^{K}$ for some unspecified $K$. The nondegeneracy condition is of course that $\langle\zeta, \mu\rangle=0$ for all $\zeta$ implies $\mu=0$. By elementary linear algebra one can always choose coordinates such that

$$
\langle\zeta, \zeta\rangle=\left|\zeta_{1}\right|^{2}+\ldots+\left|\zeta_{a}\right|^{2}-\left|\zeta_{a+1}\right|^{2}-\ldots-\left|\zeta_{a+b}\right|^{2},
$$

and $a+b=K$. Thus $Q(a, b)$ is the level set given by $\{\zeta:\langle\zeta, \zeta\rangle=1\}$.

Let $H(n, d)$ denote the complex vector space of homogeneous polynomials of degree $d$ in $n$ variables. One can think of $H(n, d)$ as the space of sections of the $d$-th tensor power of the hyperplane section bundle over complex projective space $\mathbf{P}_{n-1}$, but we will not require this interpretation here. Let $\mathcal{L}\left(\mathbf{C}^{N}, H(n, d)\right)$ denote the vector space of linear mappings from $\mathbf{C}^{N}$ to $H(n, d)$. After choosing bases we can think of elements of $\mathcal{L}\left(\mathbf{C}^{N}, H(n, d)\right)$ as matrices with $N$ columns and $\left(\begin{array}{c}n+d-1 \\ d\end{array}\right)$ rows.

We are now ready to compute $X_{f}$.

Theorem 1. Let $\langle$,$\rangle denote both a nondegenerate Hermitian form on \mathbf{C}^{n}$ and one on $\mathbf{C}^{N}$. Let $M$ denote the hyperquadric in $\mathbf{C}^{n}$ defined by $\{z:\langle z, z\rangle=1\}$, and let $M^{\prime}$ be the hyperquadric in $\mathbf{C}^{N}$ defined by $\{\zeta:\langle\zeta, \zeta\rangle=1\}$. Let $f$ be a rational function such that $f(M) \subset M^{\prime}$. Let d denote the degree of the numerator of $f$ when $f$ is reduced 
to lowest terms. Then there is a polynomial mapping $w \mapsto C(w) \in \mathcal{L}\left(\mathbf{C}^{N}, H(n, d)\right)$ such that

$$
X_{f}=\{(w, \zeta): \zeta=f(w)+\operatorname{Kernel}(C(w))\}
$$

Proof. By (9) we have

$$
X_{f}=\{(w, \zeta):\langle f(z), \zeta\rangle=\langle f(z), f(w)\rangle \text { when }\langle z, w\rangle=1\} .
$$

By linearity of the form we have

$$
X_{f}=\{(w, \zeta):\langle f(z), \zeta-f(w)\rangle=0 \text { when }\langle z, w\rangle=1\} .
$$

We are assuming that $f=\frac{p}{q}$ is rational, and we may assume that $f$ is reduced to lowest terms. Write $d$ for the degree of $p$. In case $M$ and $M^{\prime}$ are spheres, it is easy to see that the degree of $p$ must be at least the degree of $q$.

We multiply the equation in (15) by $q(z)$ to obtain

$$
X_{f}=\{(w, \zeta):\langle p(z), \zeta-f(w)\rangle=0 \text { when }\langle z, w\rangle=1\} .
$$

We may write $p=\sum_{j=\nu}^{d} p_{j}$, where each $p_{j}$ is homogeneous of degree $j$. Define a homogeneous polynomial $T_{w}: \mathbf{C}^{\mathbf{n}} \rightarrow \mathbf{C}^{N}$ by

$$
T_{w}(z)=\sum_{j=\nu}^{d} p_{j}(z)\langle z, w\rangle^{d-j} .
$$

Since $\langle z, w\rangle=1$ in (16), we can rewrite (16) using (17). We obtain

$$
X_{f}=\left\{(w, \zeta):\left\langle T_{w}(z), \zeta-f(w)\right\rangle=0 \text { when }\langle z, w\rangle=1\right\} .
$$

We define $C(w)$ by the formula:

$$
\overline{C(w)(\mu)}(z)=\left\langle T_{w}(z), \mu\right\rangle .
$$

It is evident that $\mu \mapsto C(w)(\mu)$ is linear in $\mu$, and that its output is a homogeneous polynomial of degree $d$ in $z$.

Fix $w \neq 0$. By (18) and (19) the homogeneous polynomial

$$
z \mapsto\left\langle T_{w}(z), \zeta-f(w)\right\rangle=\overline{C(w)(\zeta-f(w))}(z)
$$

vanishes on $\langle z, w\rangle=1$. For $\lambda \neq 0$, we see from homogeneity that the polynomial in (20) vanishes when $\langle z, w\rangle=\lambda$. By continuity it vanishes for all $z$. For $w \neq 0$, we conclude that $(w, \zeta) \in X_{f}$ if and only if

$$
C(w)(\zeta-f(w))=0 .
$$

Thus $(w, \zeta) \in X_{f}$ if and only if $\zeta-f(w)$ is in the kernel of $C(w)$, and hence (14) holds.

Theorem 1 has several immediate corollaries. In each of these corollaries we assume the hypotheses from the theorem without stating them explicitly. It is useful also because the computations can be done so easily. In the case of spheres [D4] 
provides an integral formula for $C(w)$. Before turning to the corollaries we note that the formula for $C(w)$ depends only upon the numerator of $f$. This superficially strange fact becomes sensible when one realizes that the numerator determines the denominator.

Corollary 1. $X_{f}$ is an affine space.

Corollary 2. $X_{f}$ equals the graph of $f$ if and only if $C(w)$ is injective for all $w$ (here $w \neq 0$ ). Also, $X_{f}$ is an affine bundle if and only if the kernel of $C(w)$ has constant dimension.

Corollary 3. If $f$ is homogeneous, then $C(w)$ is independent of $w$, and consequently $X_{f}$ is an affine bundle. If also the target dimension is minimal, then $X_{f}$ is the graph of $f$. If $f$ is not homogeneous, then $C(w)$ depends on $w$. In this case $X_{f}$ is generally not a bundle, but for some $f$ it is a bundle.

Proof. It follows from (17) and (19) that the map $w \mapsto C(w)$ is a polynomial in $w$ of degree equal to $d-\nu$, where $d$ is the degree of the numerator $p$ of $f$ and $\nu$ is the order of vanishing of $p$. In particular when $f$ is homogeneous, $C(w)$ is independent of $w$, and its kernel is independent of $w$, and hence $X_{f}$ is an affine bundle. It is not hard to see that the target dimension $N$ is minimal for a homogeneous polynomial mapping of degree $d$ precisely when $N=\left(\begin{array}{c}n+d-1 \\ d\end{array}\right)$. See Theorem 2. In this case the map $C(w)$ is an isomorphism, and thus its kernel is trivial. When $f$ is not homogeneous, $C(w)$ depends on $w$, and examples below show that both possibilities occur even for polynomial $f$.

Corollary 4. Let $f: \mathbf{C}^{n} \rightarrow \mathbf{C}^{N}$ be a polynomial mapping of degree $d$, and suppose $f(M) \subset M^{\prime}$. If $N>\left(\begin{array}{c}n+d-1 \\ d\end{array}\right)$, then $X_{f}$ strictly contains the graph of $f$. Furthermore, for each $w$, the dimension of the fibre over $w$ is at least $N-\left(\begin{array}{c}n+d-1 \\ d\end{array}\right)$.

Proof. The image of $C(w)$ is a subspace of $H(n, d)$, and hence its dimension is at most $\left(\begin{array}{c}n+d-1 \\ d\end{array}\right)$. By elementary linear algebra we obtain

$$
\operatorname{dim}(\operatorname{Kernel}(C(w)))=N-\operatorname{dim}(\operatorname{Image}(C(w))) \geq N-\left(\begin{array}{c}
n+d-1 \\
d
\end{array}\right) \cdot \mathbf{\square}
$$

Since the nullity of a linear mapping depends lower semi-continuously on parameters, the dimension of the fibre of $X_{f}$ over $w$ depends lower-semi-continuously on $w$. Thus the exceptional points are where the fibre dimension increases. Since the entries of the matrix $C(w)$ are polynomials in $w$, we see also that $X_{f}$ is itself an algebraic variety defined by the system of polynomial equations

$$
C(w)(q(w) \zeta-p(w))=0
$$

where $f=\frac{p}{q}$.

We next give some examples which illustrate some of the possibilities for $X_{f}$.

ExAmple 2. Put $f\left(z_{1}, z_{2}\right)=\left(z_{1}, z_{1} z_{2}, z_{1} z_{2}^{2}, z_{2}^{3}\right)$. Then $f$ maps the sphere $S^{3}$ to the sphere $S^{7}$. This mapping results from applying two restricted tensor product operations. See Section 2 and [D3]. We find $X_{f}$ by finding $C(w)$ and using Theorem 1. After homogenizing and choosing bases we obtain 


$$
C(w)=\left(\begin{array}{cccc}
w_{1}^{2} & 0 & 0 & 0 \\
2 w_{1} w_{2} & w_{1} & 0 & 0 \\
w_{2}^{2} & w_{2} & 1 & 0 \\
0 & 0 & 0 & 1
\end{array}\right)
$$

It is evident that the kernel is trivial for $w_{1} \neq 0$, and hence the fibre over such $w$ is simply $f(w)$. For $w_{1}=0$, however, the kernel is two-dimensional, with basis given by $\left(1,0,-w_{2}^{2}, 0\right)$ and $\left(0,1,-w_{2}, 0\right)$.

Example 3. Here we map the sphere $Q(2,0)$ in $\mathbf{C}^{2}$ to the sphere $Q(5,0)$ in $\mathbf{C}^{5}$. With $f$ defined by $(25)$, it is easy to check that $f(Q(2,0)) \subset Q(5,0)$.

$$
f(z)=\left(z_{1}^{4}, 2 z_{1}^{3} z_{2}, \sqrt{6} z_{1}^{2} z_{2}^{2}, 2 z_{1} z_{2}^{3}, z_{2}^{4}\right) .
$$

The mapping $f$ is invariant under the cyclic unitary group $G$ of order four generated by multiplication by $i$, and it thus induces a CR mapping from $S^{3} / G$ to $S^{9}$. This example should be contrasted with Example 4, where a different representation of a cyclic group of order four yields a rather different result. Using Corollary 3 , we obtain $C(w)$ :

$$
C(w)=\left(\begin{array}{ccccc}
1 & 0 & 0 & 0 & 0 \\
0 & 2 & 0 & 0 & 0 \\
0 & 0 & \sqrt{6} & 0 & 0 \\
0 & 0 & 0 & 2 & 0 \\
0 & 0 & 0 & 0 & 1
\end{array}\right) .
$$

From (26) we see that $X_{f}$ is the graph of $f$. Nothing unusual happens at $w=0$.

Example 4 . Here we map the sphere $Q(2,0)$ in $\mathbf{C}^{2}$ to the hyperquadric $Q(3,1)$ in $\mathbf{C}^{4}$. Define $f$ by (27):

$$
f(z)=\left(z_{1}^{4}, z_{2}^{4}, 2 z_{1} z_{2}, \sqrt{2} z_{1}^{2} z_{2}^{2}\right) .
$$

Here $f$ is invariant under the cyclic unitary group $\Gamma$ of order four generated by $\left(z_{1}, z_{2}\right) \mapsto\left(i z_{1},-i z_{2}\right)$. Then $f\left(S^{3}\right) \subset Q(3,1)$, and $f$ induces a CR mapping from $S^{3} / \Gamma$ to $Q(3,1)$. Using Theorem 1 we compute the matrix $C(w)$, obtaining

$$
C(w)=\left(\begin{array}{cccc}
1 & 0 & 0 & 0 \\
0 & 0 & 2 w_{1}^{2} & 0 \\
0 & 0 & 4 w_{1} w_{2} & -\sqrt{2} \\
0 & 0 & 2 w_{2}^{2} & 0 \\
0 & 1 & 0 & 0
\end{array}\right) .
$$

From (28) it is evident that $X_{f}$ is the graph of $f$; the only point where the kernel of $C(w)$ is not trivial is the origin, which is excluded from the discussion because the equation $\langle z, w\rangle=1$ is vacuous for $w=0$.

We next consider the special point $w=0$ in the case of mappings between hyperquadrics, leading to the definition of the extended variety $Y_{f}$. Because the condition $\langle z, w\rangle=1$ is vacuous when $w=0$, the point $w=0$ is not considered in the definition of $X_{f}$. On the other hand, $C(w)$ (as a matrix of polynomials in $w$ ) makes perfectly good sense at $w=0$. We therefore extend the variety $X_{f}$ by including also the points

$$
(0, f(0)+\operatorname{Kernel}(C(0)) .
$$


For clarity we write $Y_{f}$ for the resulting union.

Definition 2. Assume the set-up from Theorem 1. The extended variety $Y_{f}$ is the subset of Domain $(f) \times$ Target $(f)$ defined by

$$
Y_{f}=\{(w, \zeta): \zeta=f(w)+\operatorname{Kernel}(C(w))\} .
$$

By Theorem 1, $Y_{f}=X_{f} \cup(0, f(0)+\operatorname{Kernel}(C(0)))$.

We can easily interpret the new fibre. By the proof of Theorem 1, the matrix $C(0)$ has maximum rank if and only if the highest order part (terms of order $d$ ) of the numerator $p$ of $f$ includes all monomials of degree $d$. In order that $Y_{f}$ be a bundle, the dimension of the fibre over $w=0$ must be the same as at other points. For this to hold it is necessary that the highest order part of $p$ contain all monomials of that degree. In Example 4, although $X_{f}$ is the graph of $f, Y_{f}$ is not the graph of $f$. The fibre over $w=0$ is one-dimensional.

We summarize these remarks in the next proposition.

Proposition 1. Let $f=\frac{p}{q}$ be a rational mapping such that $f(M) \subset M^{\prime}$ for nondegenerate hyperquadrics. Define $C(w)$ by (19). The algebraic subvariety $Y_{f}$ defined by (31) then extends the definition of $X_{f}$ to include the exceptional point $w=0$.

$$
Y_{f}=\{(w, \zeta): C(w)(q(w) \zeta-p(w))=0\} .
$$

Example 5. Define $f: \mathbf{C}^{2} \rightarrow \mathbf{C}^{5}$ by $f\left(z_{1}, z_{2}\right)=\left(z_{1}^{5}, \sqrt{5} z_{1}^{3} z_{2}, \sqrt{5} z_{1} z_{2}^{2}, z_{2}^{5}\right)$. Then $f\left(S^{3}\right) \subset S^{7}$. We compute $C(w)$ and hence $Y_{f}$.

$$
C(w)=\left(\begin{array}{cccc}
1 & 0 & 0 & 0 \\
0 & \sqrt{5} w_{1} & 0 & 0 \\
0 & \sqrt{5} w_{2} & \sqrt{5} w_{1}^{2} & 0 \\
0 & 0 & 2 \sqrt{5} w_{1} w_{2} & 0 \\
0 & 0 & \sqrt{5} w_{2}^{2} & 0 \\
0 & 0 & 0 & 1
\end{array}\right) .
$$

From (32) it follows that the fibre over $w$ is $f(w)$, at all points except $w=0$, where the fibre is two-dimensional. Thus $X_{f}$ is the graph of $f$, whereas $Y_{f}$ is not. The mapping $f$ is group-invariant under a cyclic unitary group of order five, and thus $f$ induces a map from a Lens space to a sphere.

We close this section with a rational example. This example is interesting partly because it maps $Q(1,1)$ to the sphere $Q(2,0)$. Also any point where $w_{1}=0$ must be excluded because $f$ is not holomorphic there.

EXAmple 6. Let $f(z)=\left(\frac{1}{z_{1}}, \frac{z_{2}}{z_{1}}\right)$. Then $f$ is holomorphic near $Q(1,1)$. Furthermore $f(Q(1,1)) \subset Q(2,0)$ since $\left|\frac{1}{z_{1}}\right|^{2}+\left|\frac{z_{2}}{z_{1}}\right|^{2}=1$ on $\left|z_{1}\right|^{2}-\left|z_{2}\right|^{2}=1$. Using Theorem 1 we compute $C(w)$ to obtain

$$
C(w)=\left(\begin{array}{cc}
w_{1} & 0 \\
-w_{2} & 1
\end{array}\right)
$$

The kernel of $C(w)$ is trivial except where $w_{1}=0$, but such points are not in the domain of $f$. Thus $X_{f}$ is the graph of $f$. 
2. Homogeneous mappings between hyperquadrics. In this section we consider the homogeneous case in more detail. In Theorem 2 we determine all homogeneous polynomial mappings $f$ between hyperquadrics and with minimal target dimension. It follows from Corollary 3 that $Y_{f}$ equals the graph of $f$ in this case.

Let $\langle$,$\rangle be a nondegenerate Hermitian form on \mathbf{C}^{N}$; there are integers $a, b$ and coordinates such that

$$
\langle\zeta, \zeta\rangle=\sum_{j=1}^{a}\left|\zeta_{j}\right|^{2}-\sum_{j=a+1}^{a+b}\left|\zeta_{j}\right|^{2}
$$

We let $U(a, b)$ denote the group of linear transformations $L: \mathbf{C}^{N} \rightarrow \mathbf{C}^{N}$ preserving the form in (33). Thus $L \in U(a, b)$ if and only if $\langle L \zeta, L \eta\rangle=\langle\zeta, \eta\rangle$ for all $\zeta$ and $\eta$.

We begin with a simple way to construct homogeneous polynomial mappings $f: \mathbf{C}^{n} \rightarrow \mathbf{C}^{N}$ such that $\langle f(z), f(z)\rangle=1$ (for an appropriate Hermitian form) on $\langle z, z\rangle=1$. Given the form $\langle z, w\rangle$ on $\mathbf{C}^{n}$ and a positive integer $d$, the expression $\langle z, w\rangle^{d}$ is a Hermitian symmetric bihomogeneous polynomial. There are unique nonnegative integers $a$ and $b$ and (not unique) linearly independent homogeneous polynomials $A_{j}$ and $B_{j}$ of degree $d$ for which we can write

$$
\langle z, w\rangle^{d}=\sum_{j=1}^{a} A_{j}(z) \overline{A_{j}(w)}-\sum_{j=a+1}^{a+b} B_{j}(z) \overline{B_{j}(w)}
$$

The right-hand side of (34) can be written $\langle f(z), f(w)\rangle$ with $\langle$,$\rangle as in (33) for the$ homogeneous polynomial mapping $f=A \oplus B$. Then $\langle f(z), f(w)\rangle=1$ on $\langle z, w\rangle=1$. This naive idea combines with linear algebra to provide all homogeneous examples.

We mention in passing that when the form on $\mathbf{C}^{n}$ is positive definite, the form in (34) will also be positive definite and thus $b=0$. In general the form in (34) will have many negative eigenvalues. For $n \geq 2$ this number $b$ must grow as $d$ grows. The next result generalizes a result for the sphere ([D2], $[\mathrm{R}])$ that plays a crucial role in [D1] and [D3].

THEOREM 2. Let $\langle$,$\rangle denote nondegenerate Hermitian forms on both \mathbf{C}^{n}$ and $\mathbf{C}^{N}$. Let $f: \mathbf{C}^{n} \rightarrow \mathbf{C}^{N}$ be a homogeneous polynomial mapping of degree $d$ such that $\langle f(z), f(z)\rangle=1$ whenever $\langle z, z\rangle=1$. Then, for all $z$ and $w$,

$$
\langle f(z), f(w)\rangle=\langle z, w\rangle^{d}
$$

Also, $N \geq\left(\begin{array}{c}n+d-1 \\ d\end{array}\right)$. Furthermore, if $g$ and $f$ both satisfy these hypotheses and $N=$ $\left(\begin{array}{c}n+d-1 \\ d\end{array}\right)$, then there is an $L \in U(a, b)$ such that $g=L f$. Finally, write

$$
f(z)=\sum_{\alpha} C_{\alpha} z^{\alpha}
$$

The coefficient vectors $C_{\alpha}$ then satisfy $\left\langle C_{\alpha}, C_{\beta}\right\rangle=0$ for $\alpha \neq \beta$ and $\left\langle C_{\alpha}, C_{\alpha}\right\rangle= \pm\left(\begin{array}{l}d \\ \alpha\end{array}\right)$.

Proof. Suppose that $\langle f(z), f(z)\rangle=1$ whenever $\langle z, z\rangle=1$. We polarize this condition and conclude that $\langle f(z), f(w)\rangle=1$ whenever $\langle z, w\rangle=1$. Next suppose that $\langle z, w\rangle=\lambda \neq 0$. Then $\left\langle\frac{z}{\lambda}, w\right\rangle=1$, so we conclude that

$$
\left\langle f\left(\frac{z}{\lambda}\right), f(w)\right\rangle=1=\left\langle\frac{z}{\lambda}, w\right\rangle^{d} .
$$


Both $f$ and $\langle,\rangle^{d}$ are homogeneous of degree $d$ in $z$, so (37) yields (35) for all $z$ and $w$ such that $\langle z, w\rangle \neq 0$. By continuity, (35) holds for all $z$ and $w$, giving the first conclusion.

Before considering the second conclusion we write (36) for some coefficient vectors $C_{\alpha}$. Suppose that the form on $\mathbf{C}^{n}$ has $k$ positive eigenvalues. We may write

$$
\langle z, w\rangle=\sum \pm(j) z_{j} \bar{w}_{j},
$$

where $\pm(j)=1$ for $j \leq k$ and $\pm(j)=-1$ for $j>k$. Expanding (38) by the multinomial theorem yields

$$
\langle z, w\rangle^{d}=\sum_{\alpha}\left(\begin{array}{l}
d \\
\alpha
\end{array}\right)(z \bar{w})^{\alpha} a(\alpha)
$$

where each $a(\alpha)= \pm 1$.

Plugging (39) and (36) into (35) yields

$$
\sum_{\alpha, \beta}\left\langle C_{\alpha}, C_{\beta}\right\rangle z^{\alpha} \bar{w}^{\beta}=\langle z, w\rangle^{d}=\sum_{\alpha}\left(\begin{array}{l}
d \\
\alpha
\end{array}\right)(z \bar{w})^{\alpha} a(\alpha) .
$$

Varying $z$ and $\bar{w}$ separately in (40) and equating coefficients gives the formulas for the $\left\langle C_{\alpha}, C_{\beta}\right\rangle$. Since $\left\langle C_{\alpha}, C_{\alpha}\right\rangle \neq 0$ for all $\alpha$, all the coefficient vectors are not zero. Since $\left\langle C_{\alpha}, C_{\beta}\right\rangle=0$ for $\alpha \neq \beta$ and the Hermitian form is nondegenerate, the coefficient vectors are linearly independent. Thus $N$ is at least as large as the dimension of $H(n, d)$, which is $\left(\begin{array}{c}n+d-1 \\ d\end{array}\right)$.

Finally let $g$ be another such mapping, with coefficient vectors $D_{\alpha}$, and assume that $N$ is minimal. Using (40) again we conclude that $\left\langle D_{\alpha}, D_{\beta}\right\rangle=\left\langle C_{\alpha}, C_{\beta}\right\rangle$ for all multi-indices $\alpha$ and $\beta$. Since all inner products are preserved, and the linear independence condition holds for each set of vectors, we can define $L \in U(a, b)$ by setting $L\left(C_{\alpha}\right)=D_{\alpha}$. It follows that $g=L f$. $\mathrm{Q}$

Corollary 5. Let $f: \mathbf{C}^{n} \rightarrow \mathbf{C}^{N}$ be a homogeneous polynomial mapping of degree d. Suppose $\langle f(z), f(z)\rangle=1$ on $\langle z, z\rangle=1$. Then $Y_{f}$ is the graph of $f$ if and only if $N=\left(\begin{array}{c}n+d-1 \\ d\end{array}\right)$.

Proof. For any polynomial of degree $d$ Corollary 4 implies, when $N>\left(\begin{array}{c}n+d-1 \\ d\end{array}\right)$, that $X_{f}$ and hence $Y_{f}$ strictly contain the graph of $f$. If also $f$ is homogeneous, then $Y_{f}$ equals the graph of $f$ if and only if the target dimension is minimal by Corollary 3. By Theorem 2, the target dimension $N$ is minimal if and only if $N=\left(\begin{array}{c}n+d-1 \\ d\end{array}\right)$.

Corollary 5 generalizes Example 1. In Example 1 any excess in target dimension for a linear map $L$ is revealed as the fibre dimension of $X_{L}$. In Corollary 5 we see the same result in the homogeneous case.

Given a homogeneous polynomial map $f$ such that $f(Q(a, b)) \subset Q\left(a^{\prime}, b^{\prime}\right)$, we can compose $f$ with a linear map $L$ into higher dimensions that sends $Q\left(a^{\prime}, b^{\prime}\right)$ into $Q\left(a^{\prime \prime}, b^{\prime \prime}\right)$ for appropriate $a^{\prime \prime}$ and $b^{\prime \prime}$. Then $g=L \circ f$ maps $Q(a, b)$ to $Q\left(a^{\prime \prime}, b^{\prime \prime}\right)$. We can detect such compositions of maps because $Y_{g}$ must have positive dimensional fibres. Using these ideas it is possible to combine Theorem 2 with the rigidity results in $[\mathrm{BH}]$.

It is also easy to construct general polynomial examples. In a future paper we will describe a procedure for homogenization analogous to the orthogonal homogenization procedure from [D1] and [D2] stated in Theorem 3 below. 
Let $u$ be a polynomial in one variable with $u(1)=1$. We may expand $u(\langle z, w\rangle)$, and choose signs such that (41) holds for some polynomial mapping $f$ and appropriate form on the target of $f$ :

$$
u(\langle z, w\rangle)=\langle f(z), f(w)\rangle .
$$

Using this method we can construct as many examples as we wish of polynomial mappings between hyperquadrics, as long as we allow the form in the target space to have enough negative eigenvalues. Again we observe the complexity issue. See [BH] and [EHZ1] for what happens when one restricts the number of negative eigenvalues.

The homogeneous polynomial mappings taking spheres to spheres play a key role in the classification problem and have many additional interesting properties. We can use them to study arbitrary polynomial maps taking spheres to spheres. We do so by considering the volume of the image of the unit ball $B_{n}$ under such maps.

Let $f: B_{n} \rightarrow \mathbf{C}^{N}$ be a holomorphic mapping. Recall that the volume (with multiplicity counted) of the image of the ball $B_{n}$ under $f$ is given by

$$
V(f)=\sum \int_{B_{n}}\left|J\left(f_{j_{1}}, \ldots, f_{j_{n}}\right)\right|^{2} d V=\int_{B_{n}} H(f) d V .
$$

In (42) the sum is taken over all choices of $n$-tuples of components of $f$, and $J$ denotes the Jacobian determinant. In the second term $H(f)$ denotes the determinant of the complex Hessian of $f$. Using wedge products one can easily verify that the two integrands in (42) are the same. In case $f$ is homogeneous of degree $d$, the volume turns out to be $\frac{d^{n} \pi^{n}}{n !}$, independent of the target dimension. See [D1].

In [D2] and [D3] the homogeneous maps between spheres are used to classify all polynomial maps between spheres, via a tensor product operation. We briefly sketch these ideas. Let $H_{m}$ denote a homogeneous polynomial mapping of degree $m$ whose components are linearly independent and for which

$$
\left\|H_{m}(z)\right\|^{2}=\|z\|^{2 m} .
$$

It is evident from (43) that $H_{m}$ maps the sphere in its domain to the sphere in its target. Its components are linearly independent and they must also span the space of homogeneous polynomials of degree $m$ in $n$ variables for (43) to hold. Theorem 2 therefore implies that $H_{m}$ is determined up to a unitary map by (43). (See also [D2] and [R].) Corollary 3 guarantees that the $X$-variety for $H_{m}$ is its graph. Each polynomial mapping of degree $m$ between spheres can be "tensored" into $H_{m}$. Each tensor operation increases the volume of the image of the ball.

Let $A$ be a subspace of $\mathbf{C}^{N}$ with orthogonal complement $A^{\perp}$. Let $\pi$ denote the orthogonal projection onto $A$. Given a function $p$ with values in $\mathbf{C}^{N}$ we naturally write

$$
p=(1-\pi)(p) \oplus \pi(p) .
$$

Let $g$ be a function with the same domain as $p$ and with values in some $\mathbf{C}^{k}$. We define a mapping $E_{A}(p, g)$ by

$$
E_{A}(p, g)=(1-\pi)(p) \oplus(\pi(p) \otimes g) .
$$

In case $p$ and $g$ map the sphere to the sphere, a simple calculation shows that $E_{A}(p, g)$ also does. Notice that the target dimension of $E_{A}(p, g)$ is $(N-a)+a k$, where $a=\operatorname{dim}(A)$ (hence $N-a=\operatorname{dim}\left(A^{\perp}\right)$ ) and $k$ is the dimension of the target of $g$. 
When $g$ is the identity mapping $z$, the restricted tensor product operation $p \mapsto$ $E_{A}(p, g)$ is a kind of orthogonal homogenization, closely related to the homogenization part of the proof of Theorem 1. The inverse operation sending $E_{A}(p, g) \mapsto p$ is a kind of orthogonal dehomogenization. When the subspace $A$ is one-dimensional, say the span of the vector $e_{1}$, we have

$$
E_{A}(f, z)=\left(z_{1} f_{1}, \ldots, z_{n} f_{1}, f_{2}, \ldots, f_{N}\right)
$$

The inverse operation replaces the right-hand side of (46) with $f$.

The author [D2] and [D3]) obtained the following result as part of the classification problem for proper polynomial mappings between balls.

TheOREM 3. Let $p: \mathbf{C}^{n} \rightarrow \mathbf{C}^{N}$ be a polynomial mapping of degree $m$ such that $p\left(S^{2 n-1}\right) \subset S^{2 N-1}$. Let $H_{m}$ satisfy (43). Then there is an integer $N^{\prime}$, subspaces $A_{0}$, $\ldots, A_{m}$ of $\mathbf{C}^{N^{\prime}}$, tensor products $E_{0}, \ldots E_{m}$, and a linear mapping $L: \mathbf{C}^{N^{\prime}} \rightarrow \mathbf{C}^{N(n, m)}$ such that

$$
H_{m}=L \prod_{j=0}^{m} E_{j}(p) .
$$

Each tensor product $E_{j}$ is of the form $E_{A_{j}}(f, z)$ for the corresponding subspace $A_{j}$.

Let $f: B_{n} \rightarrow \mathbf{C}^{N}$ be a holomorphic mapping for which the volume $V_{f}$ of the image of the ball under $f$ is finite. By a monotonicity result from [D1], the volume of the image of the ball under $E_{A}(f, z)$ exceeds $V_{f}$ unless $\pi_{A}(f)=0$. By combining this result with Theorem 3 we obtain a characterization of the homogeneous polynomial mappings between spheres as extremals for volumes.

THEOREM 4. [D1] Let $p: \mathbf{C}^{n} \rightarrow \mathbf{C}^{N}$ be a polynomial of degree $m$, and suppose that $p\left(S^{2 n-1}\right) \subset S^{2 N-1}$. Then the volume (with multiplicity counted) of the image of the unit ball under $p$ is at most $\frac{m^{n} \pi^{n}}{n !}$, and equality occurs if and only if $p$ is homogeneous.

Theorem 2 has a nice geometric interpretation. A homogeneous polynomial mapping of degree $m$ covers the ball $m$ times, introducing a factor of $m^{n}$ into the volume. Lower order terms mean that the multiplicity is less than $m$ at some points, so the full impact of this factor is not felt. We compute the volumes $V$ of the images of the two-ball under the three maps in (48) to illustrate this idea.

$$
\begin{gathered}
\left(z_{1}, z_{2}\right) \mapsto\left(z_{1}, z_{2}\right) \cdot V=\frac{\pi^{2}}{2} \\
\left(z_{1}, z_{2}\right) \mapsto\left(z_{1}, z_{1} z_{2}, z_{2}^{2}\right) \cdot V=\frac{7 \pi^{2}}{6} \\
\left(z_{1}, z_{2}\right) \mapsto\left(z_{1}^{2}, \sqrt{2} z_{1} z_{2}, z_{2}^{2}\right) \cdot V=2 \pi^{2} .
\end{gathered}
$$




\section{REFERENCES}

[BRE] M. S. Baouendi, P. Ebenfelt, and L. P. Rothschild, Real Submanifolds in Complex Space and Their Mappings, Princeton Math. Series 47, Princeton Univ. Press, 1999.

[BH] M. S. BaOUendi And XiaOjun HuAng, Super-rigidity for holomorphic mappings between hyperquadrics with positive signature, J. Diff. Geom., 69:2 (2005), pp. 379-398.

[CD1] David W. Catlin and John P. D'Angelo, A stabilization theorem for Hermitian forms and applications to holomorphic mappings, Math Research Letters, 3 (1996), pp. 149166.

[CD2] David W. Catlin and John P. D'Angelo, An isometric imbedding theorem for holomorphic bundles, Math Research Letters, 6 (1999), pp. 1-18.

[CS] J. A. Cima And T. J. Suffridge, Boundary behavior of rational proper maps, Duke Math J., 60:1 (1990), pp. 125-138.

[D1] John P. D'Angelo, A monotonicity result for volumes of holomorphic images, Michigan Math J., 54 (2006), pp. 623-646.

[D2] John P. D'Angelo, Several Complex Variables and the Geometry of Real Hypersurfaces, CRC Press, Boca Raton, 1993.

[D3] John P. D'Angelo, Proper holomorphic mappings, positivity conditions, and isometric imbedding, J. Korean Math Society, (2003), pp. 1-30.

[D4] John P. D'Angelo, Homogenization, reflection, and the X-variety, Indiana U. Math J., 52:5 (2003), pp. 1113-1132.

[D5] John P. D'Angelo, Inequalities from Complex Analysis, Carus Mathematical Monograph Number 28, Mathematical Association of America, 2002.

[DKR] John P. D'Angelo, Šimon Kos, And Emily Riehl, A sharp bound for the degree of proper monomial mappings between balls, The Journal of Geometric Analysis, 13:4 (2003), pp. 581-593.

[DF] K. Diederich and J. E. Fornaess, Proper holomorphic mappings between real-analytic pseudoconvex domains in $\mathbf{C}^{n}$, Math Ann., 282 (1988), pp. 681-700.

[DW] K. Diederich AND S. M. WeBSTER, A reflection principle for degenerate real hypersurfaces, Duke Math. J, 47 (1980), pp. 835-843.

[EHZ1] Peter Ebenfelt, Xiaojun Huang, Dmitri Zaitsev, Rigidity of CR-immersions into spheres, Comm. Anal. Geom, 12:3 (2004), pp. 631-670.

[EhZ2] Peter Ebenfelt, Xiaojun Huang, Dmitri Zaitsev, The equivalence problem and rigidity for hypersurfaces embedded in hyperquadrics, Amer. J. Math, 127:1 (2005), pp. 169-191.

[Fa1] J. FARAN, Linearity of proper holomorphic mappings in the low codimension case, J. Diff. Geom., 24 (1986), pp. 15-17.

[Fa2] J. FARAn, Maps from the two-ball to the three-ball, Inventiones Math., 68 (1982), pp. 441475.

[F1] Franc Forstneric, Extending proper holomorphic mappings of positive codimension, Inventiones Math, 95 (1989), pp. 31-62.

[F2] Franc Forstneric, Proper rational maps: A survey, pp. 297-363 in Several Complex Variables: Proceedings of the Mittag-Leffler Institute, 1987-1988, Mathematical Notes 38, Princeton Univ. Press, Princeton, 1993.

$[\mathrm{H}]$ X. HuAng, On a linearity problem for proper maps between balls in complex spaces of different dimensions, J. Diff. Geom., 51:1 (1999), pp. 13-33.

[HJ] X. Huang, X., ANd S. JI, Mapping $B_{n}$ into $B_{2 n-1}$, Inventiones Math., 145 (2001), pp. 219250.

[LaM] B. Lamel and N. Mir, Parametrization of local CR automorphisms by finite jets and applications, J.A.M.S. (to appear).

$[\mathrm{P}]$ S. I. PINCUK, On the analytic continuation of holomorphic mappings, Math USSR-Sb. 27 (1975), pp. 375-392.

[R] Walter Rudin, Homogeneous polynomial maps, Nederl. Akad Wetensch. Indag. Math., 46 (1984), pp. 55-61.

[W] S. M. WeBSTER, On the mapping problem for real algebraic hypersurfaces, Inventiones Math., 43 (1977), pp. 53-68. 